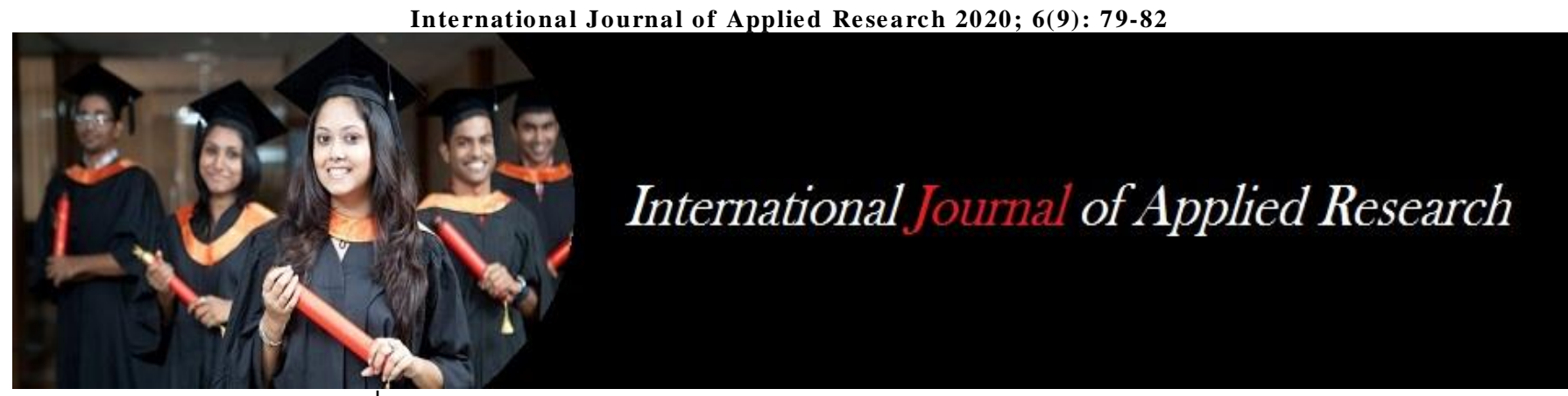

ISSN Print: 2394-7500 ISSN Online: 2394-5869 Impact Factor: 5.2

IJAR 2020; 6(9): 79-82 www.allresearchjournal.com Received: 01-07-2020 Accepted: 06-08-2020

Chetali Paliwal PES Modern College of Physiotherapy, Jangli Maharaj Road, Shivajinagar, Pune, Maharashtra, India

Dr. Shefali Walia Indian Spinal Injury Centre Institute of Rehabilitation Sciences, Vasant Kunj, New Delhi, India
Corresponding Author: Chetali Paliwal PES Modern College of Physiotherapy, Jangli Maharaj Road, Shivajinagar, Pune, Maharashtra, India

\section{Effect of secondary cognitive task on performance of stair descent in people with idiopathic Parkinson's disease}

\author{
Chetali Paliwal and Dr. Shefali Walia
}

\section{Abstract}

Background: In people with Parkinson's disease (PD), dual tasking is difficult because of the impaired motor functions of the basal ganglia. It causes postural instability, reduction in gait speed, decreased stride length, increased festination and freezing. So it can also influence performance on stair ambulation.

Objective: To study the effect of dual tasks on stair descent.

Methods: 30 subjects were selected from various hospitals in Delhi. After taking the informed consent, the subjects were made to descend down the stairs under two conditions: stair descent only and stairs descent with secondary cognitive task. The time taken in both the activities was recorded along with the scores on Observational Stair Parameter Scoring System (OSPS).

Results: Mean Time taken by the subjects was $10.63 \pm 7.199$ seconds in stair descent only and $24.57 \pm 15.076$ seconds in stair descent with secondary cognitive task. And mean value of the OSPS

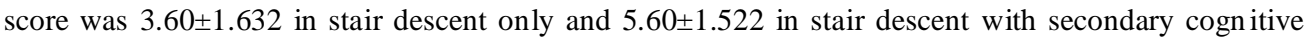
task.

Conclusion: The performance of stair descent with secondary task became more difficult than descent without the secondary task.

Keywords: Idiopathic Parkinson's disease, stair descent, secondary cognitive task

\section{Introduction}

Parkinson's disease is a chronic, progressive neuro-degenerative disorder that is pathologically defined by degeneration of the dopaminergic neurons in the substantia nigra and development of Lewy bodies in the residual dopaminergic neurons. The diagnosis of Parkinson's disease is purely clinical and relies on the presence of cardinal features of bradykinesia, rigidity, tremor, and postural instability, along with gradual symptom progression and a sustained response to levodopa therapy. Pathologic changes in Parkinson's disease may start with non-specific symptoms like hyposmia, constipation and fatigue, upto 20 years before the onset of motor symptoms. It is estimated that about $1 \%$ of population more than 60 years of age and about $4 \%$ more than 80 years of age, suffer from $\mathrm{PD}^{[1,2]}$.

PD is probably caused by complex interactions between genetic and environmental factors ${ }^{[3]}$. The male female ratio for incidence of PD in Asian population (1.0 to 1.2) was reported to be lower than worldwide spread (0.7 to 2.4$)^{[4]}$.

The diagnosis of Parkinson's disease is basically done on clinical grounds relying on the presence of its cardinal features (bradykinesia, rigidity, tremors and postural instability) along with gradual symptom progression and a sustained response to levodopa therapy ${ }^{[1]}$. Even in the early stages of disease, these people show signs of cognitive dysfunctions. Attention, executive function and memory are the most affected areas of cognitive impairments in such patients ${ }^{[5]}$. This occur due to abnormal functioning of fronto-striatal circuits ${ }^{[6]}$. There is increased dependency on cognitive control as the compensation is carried out by activation in more cortical areas. In the domain of controlled processing, due to increased pre-frontal activation the compensatory mechanism is generated, which reflects cortico-cortical and subcortico-cortical shifts of activation ${ }^{[6]}$.

In patients with Parkinson's disease quality of life is severely affected due to decline in physical activity. This is because of marked decrease in gait and balance abilities, which 
thereby causes increased fear of falling. Bradykinesia, impaired co-ordination, compromised equilibrium due to sensory issues and inflexible motor commands, causes impairments in balance. This causes poorly controlled postural adjustments to anticipate upcoming perturbations or to allow recovery from instability ${ }^{[6]}$.

People with Parkinson's disease also have marked features of gait disturbances including slow, short stepped shuffling and forward-stooped gait with asymmetrical arm swing. It includes difficulties with the execution of habitual movement sequences (like walking, turning, writing and transfers) leading to episodes of freezing and falls. Also there is difficulty seen in climbing stairs, thereby causing considerable anxiety in such patients. All these affect quality of life and participation in societal roles ${ }^{[6,7,8]}$.

During many activities of daily living, people need to perform more than one task at a time. This dual task involves the execution of a primary task, which is the major focus of attention, and a secondary task performed at the same time. In such patients, because of the impaired motor functions of the basal ganglia, dual tasking becomes difficult. The basal ganglia play a vital role in control of learned, repetitive movement sequences through their outputs to the supplementary motor area and brainstem locomotor regions. As movements become automatic they are thought to be controlled by basal ganglia, so theoretically a person can direct attention to controlling more novel or attention-demanding tasks through the use of frontal cortical regions. But in people with Parkinson's disease, normal movement pattern is generated when attention is focused on the performance; attention is thought to lead to a bypassing of the basal ganglia and the use of cortical regions to drive the outputs. In dual task situations, cortical resources may be engaged in maintaining the performance of the secondary task, leaving responsibility for regulating the performance of the more automatic task to the defective basal ganglia circuitry ${ }^{[9]}$.

People with Parkinson's disease have difficulty in performing motor tasks coupled with cognitive tasks ${ }^{[9]}$. Increased cognitive load also effect postural stability in such patients. Due to this secondary task, there is increased excursion of the centre of pressure, but with increase in complexity of task, this effect is reduced as there is overconstraining of posture, to focus attention on the cognitive task while maintaining their balance ${ }^{[11]}$.

Performance of dual tasks by these patients, causes marked gait impairments, which include reduction in gait speed and stride length, decreased symmetry and coordination between left and right steps, and increased stride to stride variability ${ }^{[10]}$. Along with gait, stair ambulation is also a component of mobility of a person. In elderly people, activity of stair ambulation revealed, more cautious behaviour during stair descent than during ascent. Also upper-body frontal plane instability was found in more number of people during stair descent ${ }^{[12]}$.

So a study is required to know, how a secondary cognitive task influence the performance of such patients on stairs, especially stair descent. Thus the aim of the study was to determine the effect of secondary cognitive tasks on performance of stair descent in people with idiopathic Parkinson's disease.

\section{Material and Methods}

It is a single group experimental study which was conducted at Indian Spinal Injuries Centre, vasant kunj, New Delhi, India. A sample of 30 subjects diagnosed with idiopathic Parkinson's disease were selected and recruited on the basis of inclusion and exclusion criteria. The Participants were recruited from the Neurology Dept. OPD of the hospital.

\subsection{Inclusion criteria}

1. Subjects diagnosed with Idiopathic Parkinson's Disease aged 31-85 years (mean age 64.5 years) ${ }^{[13]}$.

2. MMSE score $\geq 24^{[15]}$

3. Hoehn yahr score 1-3 ${ }^{[16]}$.

4. Able to ascend and descend stairs independently with/without assistive device

5. Minimum primary level education to have basic mathematical abilities

6. Subjects willing to sign the consent form to participate in the study

\subsection{Exclusion criteria}

1. Patients with any other neurological or musculoskeletal disorder which may affect gait

2. Patients with history of fall in preceding year ${ }^{[17]}$.

3. Patients with unstable or progressing medical conditions

4. Patients with sensory disturbances

5. Patients with severe or uncorrected visual deficits ${ }^{[18]}$.

6. Patients with hearing deficits

Demographic data like gender, age, height and weight, of the subjects were collected. Subjects who fulfilled the inclusion criteria were taken for the study. Procedure was adequately explained to the subjects prior taking a written consent from each one. This was followed by execution of test.

\subsection{Procedure}

To avoid fatigue, the procedure was always performed in the morning. The testing procedure includes two activities-

- Stair descent only

- Stair descent with cognitive task

In stair descent only, the participants were asked to climb down the stairs in a speed at which they could climb down most comfortably. Each participant stood at the edge of the platform and just after receiving the command "start", they would climbed down the stairs. This time was recorded by the stopwatch until both the feet touched the ground. Participants were rated on OSPS by the help of videos of the activities (recorded by the camera). During stair descent with cognitive task, participants simultaneously climb down the stairs and performed a cognitive task. A digit subtraction task was used as a cognitive task wherein each participant counted backward aloud by threes from the number 100 . The rules governing the stair descent with cognitive task were the same as those for stair descent only. No feedback was given to the participants during the performance of any of the two conditions of stair descent.

Total time taken by each participant during the two activities of stair descent was measured using a stopwatch, 
and the time was measured in seconds. Observational Stair Parameters and Scoring System measures factors like handrail use, walking style (two feet per step or step over step), sideways positioning on the stairs, medial/lateral position on the stairs, assessment of exaggerated frontal plane movement of the upper body, and hesitation at the transition region.

The complete experiment was completed in a single session of approximately one hour per patient. All the clinical outcomes obtained were recorded in the data collection form and analysis was done.

Statistical analysis was done using IBM SPSS software version 21. Paired T-test was used to find out the difference of means of time taken and OSPS score among the two conditions. A significance level of $\mathrm{p} \leq 0.05$ was fixed.

\section{Results}

The sample consisted of a total of 30 subjects with 14 males and 16 females with a mean age of $61.87 \pm 10.517$ years, mean hoehn yahr score of $1.97 \pm 0.809$, mean MMSE score of $26.90 \pm 2.074$, mean height of $169.77 \pm 6.26$ metres and mean weight of $68.48 \pm 5.98 \mathrm{~kg}$. The values of mean of time taken by the patients were $10.63 \pm 7.199$ in stair descent only, $24.57 \pm 15.076$ in stair descent with secondary cognitive task (figure 1). The mean values of the OSPS score were $3.60 \pm 1.632$ in stair descent only and $5.60 \pm 1.522$ in stair descent with secondary cognitive task (figure 2).

\section{Discussion}

This study was done to find out, whether there is an effect of secondary cognitive task on performance of stair descent in people with idiopathic Parkinson's disease.

The results show marked differences in the performance of stair descent when the subjects performed it with secondary cognitive task. The mean time taken was increased by 13.94 seconds when the secondary cognitive task was added to the stair descent task in people with Parkinson's disease. The mean value of OSPS score was increased by 2 times when the secondary cognitive task was added to the task of stair descent.

In this study the result shows that the subjects with idiopathic Parkinson's disease have demonstrated increase in the values of mean time and mean score of OSPS. Thus interference in performance of stair descent was inferred, when secondary cognitive task was undertaken with stair descent. This may be possibly explained by central nervous system processing mechanisms which were being used to perform the digit subtraction task as supported by the findings of the study conducted by O'Shea et al (2002) ${ }^{[10]}$ on dual task interference in people with Parkinson's disease. Theoretically, this requires gait to be controlled by impaired basal ganglia, which leads to increase in time taken and greater instability while performing the secondary task.

The study by J D Holmes et al. ${ }^{[11]}$ revealed that dual task interference produced increase excursion of center of pressure. This was may be because, the participants with Parkinson's disease, while performing dual task were overconstrainig their postural adjustments to prevent them from losing their balance. In such cases "posture-first principle" is applied to a pathological level, thus reducing proprioceptive feedback and may increase co-contraction. These two phenomenons diminish the individual's ability to respond to unexpected perturbations of balance. Thus it leads to increased time taken by them to complete the descent on stairs with secondary task in our study. But this overconstrainig by individuals to avoid falls causes a greater risk of falls.

A study by M Plotnik ${ }^{[21]}$ on Parkinson's disease patients states, to maintain regular walking these patients utilize their cognitive resources. So when they perform a secondary task, their cognitive resources are utilized for it, thus reducing the availability of resources for maintaining gait and hence increases the risk of fall. This again support the results in our study which shows greater instability and increased time taken by the patients with Parkinson's disease to perform the dual task in order to prevent them from falls.

When dual tasking is done, the available processing capacity is shared between the two tasks and this influences the performance of one or both the tasks ${ }^{[10]}$. Since the secondary tasks were more complicated and less familiar than the primary task, so the participants required higher attention resources to descend stairs with secondary tasks. Attention was directed more towards the secondary tasks as compared to the task of stair descent ${ }^{[19]}$. Thus the results of this study show similarities and differences with other studies conducted on patients with idiopathic Parkinson's disease.

\section{Clinical Relevance}

The data obtained in our study suggest that there is a significant effect of secondary cognitive task on the performance of stair descent in people with idiopathic Parkinson's disease. So it is advisable to avoid any such task while using stairs to prevent falls. But sometimes it is difficult to avoid dual tasks in our daily routine, so rehabilitation program for such patients should include dual task training. This will increase their confidence and reduce the risk of falls. Thereby, making them more independent and improving their quality of life.

\section{Limitations}

1. Only patients with idiopathic Parkinson's disease were selected and not the other variants of Parkinson's disease.

2. Patients having freezing episodes were not included in the study.

\section{Future Research}

Effects of different types of secondary tasks while using stairs should also be checked in Parkinson's patients. Patients with other than idiopathic Parkinson's should also be tested for dual task interference. There is also a need to conduct a study to see effects of dual tasking on freezing of gait on people with Parkinson's disease. Effect of dual tasking can also be checked on more challenging activities like crossing a road, moving in a market place, etc. Based on the results of this study, a standard protocol must be made and administered to train such patients for performing dual tasks in their activities of daily living.

\section{Conclusion}

The data obtained from the study showed that performance of stair descent with secondary cognitive task was difficult for people with idiopathic Parkinson's disease. Thus the hypothesis that there is a significant difference in performance of stair descent with secondary cognitive task in patients with idiopathic Parkinson disease holds true. So it is conclued that due to decreased physical and cognitive 
functions in people with Parkinson's disease, there is higher risk of falling during dual task performances leading to poor quality of life. So strategies should be there either to avoid dual tasking work or to train them to deal with such tasks.

\section{References}

1. John D, Gazewood D, Roxanne Richards, Karl Clebak. Parkinson disease: An Update. American Academy of Family Physicians website.

2. Wood BH, Bilclough JA, Bowron A, Walker RW. Incidence and predication of falls in Parkinson's disease: a prospective multidisciplinary study. Journal of neurology neurosurgery psychiatry. 2002; 72:721725.

3. Muslimovic D, Post B, Speeman JD. Cognitive profile of newly diagnosed Parkinson's disease. Neurology. 2005; 65:1239-1245.

4. Simon JG Lewis, Roshan Cools, Trevor W Robbins, Anja Dove. Using executive heterogeneity to explore the nature of working memory deficits in Parkinson's disease. Neuropsychologia. 2003; 41:645-654

5. Janneke Koerts, Klaus L Leenders, Wiebo H Brouwer. Cortex. 2009; 45:922-929.

6. David Conradson, Erika Franzen, Maria Hagstromer. The Effects of Highly Challenging Balance Training in Elderly With Parkinson's Disease: A Randomized Control Trial. Neurorehabilitaion and Neuro Repair, 2015, 1-10

7. Levangie PK, Norkin CC. Joint structure and function: a comprehensive analysis. FA Davis. 2001; 373:522523.

8. Samo Ribaric. Cognition and Gait Disturbances in Parkinson's Disease, Symptoms of Parkinson's Disease, Prof. Abdul Qayyum Rana (Ed.), 2011, ISBN: 978-953307-464-1.

9. Maria H Nilsson, Gun-Marie Hariz, Susan Iwarsson. Walking ability is a major contributor to fear of falling in people with Parkinson's Disease: Implications for Rehabilitation. Parkinson's disease, 2012, 713236

10. Simone O'Shea, Meg E Morris, Robert Iansek. Dual task interference during gait in people with Parkinson disease: Effects of motor versus cognitive secondary tasks. Physical Therapy Journal. 2002; 82:888-897.

11. Holmes JD, Jenkins ME, Johnson AM, Adams SG. Dual - task interference: the effects of verbal cognitive tasks on upright postural stability in Parkinson's disease. Parkinson's disease, 2010, 696492.

12. Kathryn A Hamel, Peter $R$ Cavanaugh. Stair performance in people aged 75 and older. American Geriatric Society. 2004; 52:563-567.

13. Andrew J Hughes, Susan E Daniel, Linda Kilford, Andrew J Lees. Accuracy of clinical diagnosis of idiopathic Parkinson's disease: a clinic-pathological study of 100 cases. Journal of Neurology, Neurosurgery and Psychiatry. 1992; 55:181-184.

14. Cavanaugh JT, Ellis TD, Earhart GM, Ford MP. Capturing ambulatory activity decline in Parkinson's disease. Jour Neuro Phys Ther. 2012; 36(2):51-57.

15. Folstein MF, Folstein SE, McHugh PR. "Mini-Mental State" A Practical Method For Grading The Cognitive State of Patients For The Clinician. J. Psychiatry. 1975; 12:189-198.

16. Hoehn M Yahr MD. Parkinsonism: onset, Progression and Mortality. Neurology. 1967; 17:427.
17. Nilsson MH, Hariz GM, Iwarsson S. Walking ability is a major contributor to fear of falling in people with Parkinson's disease: Implications for rehabilitation. Parkinson's disease, 2012, 713236.

18. Veronica Miyasike-daSilva, William E. Mcllroy. Does it really matter where you look when walking on stairs? Insights from a dual-task study. Plos One. 7(9):e44722.

19. Yadav M, Nayeem U Zia, Shefali Walia. Effect of Motor versus Cognitive Secondary Tasks on Performance of Stair Descent In Community Dwelling Older Adults. Activities, Adaptation and Aging. 2015; 39(1):43-55.

20. Plotnik M, Giladi N, Hausdorff JM. Bilateral coordination of gait and Parkinson's disease: the effects of dual tasking. Journal of Neurology, Neurosurgery, Psychiatry. 2009; 80:347-350

21. Abbruzzese G, Trompetto C, Marinelli L. The rationale for motor learning in Parkinson's disease. European Journal of Physical and Rehabilitation Medicine. 2009; 45:209-14. 